\title{
Osteoporosis Screening Is Often Indicated but Overlooked Prior to Rotator Cuff Repair
}

\author{
Eric J. Cotter, M.D., Emma L. Klosterman, M.D., Alec E. Winzenried, M.D., \\ Justin J. Greiner, M.D., and Brian F. Grogan, M.D.
}

\begin{abstract}
Purpose: To (1) report the percentage of patients undergoing rotator cuff repair (RCR) who were appropriately screened with dual-energy X-ray absorptiometry testing prior to surgery, if indicated, and (2) determine the percentage of patients properly prescribed osteoporosis medications within 6 months of surgery. Methods: Consecutive patients aged 50 years or older who underwent elective RCR at a single tertiary-care center over a l-year period were reviewed. Fracture risk was estimated using the Fracture Risk Assessment Tool (FRAX) with and without bone mineral density. The U.S. National Osteoporosis Foundation (NOF) criteria for screening and treatment were applied. Patients with acute fractures or revision surgery were excluded. Results: Of the 218 patients included, 129 were women (58.1\%). The mean age was $61.5 \pm 7.2$ years. One hundred thirty-one RCRs (60.1\%) occurred within 3 months of injury. A total of 69 patients (31.7\%) met the NOF criteria for bone mineral density screening. Of these patients, $23(33.3 \%)$ were appropriately screened with a dualenergy X-ray absorptiometry scan. Primary care providers initiated bone health assessment in 18 of the 23 appropriately screened patients, with orthopaedic providers initiating the workup in 3 patients (13.0\%). Thirty-two patients (14.7\%) met the NOF criteria for pharmacologic management of osteoporosis. Of these patients, 5 (15.6\%) were treated. Patients meeting the medication criteria were older $(69.1 \pm 7.7$ years vs $60.2 \pm 6.3$ years, $P<.001)$, had a lower body mass index $(28.8 \pm 6.1$ vs $31.5 \pm 6.5, P=.028)$, and had chronic rotator cuff tears $(P=.015)$. Conclusions: Patients aged 50 years or older undergoing RCR are often not appropriately screened for osteoporosis. Even when appropriately screened, only $15.6 \%$ of patients meeting the indications for pharmacologic intervention for bone health optimization were prescribed appropriate medications. Although bone health optimization may or may not affect surgical timing, patient encounters related to rotator cuff tears can be used as an opportunity for providers to initiate osteoporosis screening and treatment protocols. Level of Evidence: Level IV, therapeutic case series.
\end{abstract}

$\mathbf{O}$ steoporosis is a common condition in the United States. Wright et al. ${ }^{1}$ reported that of the 99 million U.S. citizens aged 50 years or older, 10.2 million had osteoporosis. Osteoporosis is diagnosed using the World Health Organization criteria based on dualenergy X-ray absorptiometry (DXA) screening. A bone

From the Department of Orthopedics and Rehabilitation, University of Wisconsin School of Medicine and Public Health, Madison, Wisconsin, U.S.A.

The authors report no conflicts of interest in the authorship and publication of this article. Full ICMJE author disclosure forms are available for this article online, as supplementary material.

Received August 31, 2020; accepted January 5, 2021.

Address correspondence to Eric J. Cotter, M.D., Department of Orthopedics and Rehabilitation, University of Wisconsin School of Medicine and Public Health, 1685 Highland Ave, Sixth Floor, Madison, WI 53705, U.S.A. E-mail: ecotter@uwhealth.org

(C) 2021 THE AUTHORS. Published by Elsevier Inc. on behalf of the Arthroscopy Association of North America. This is an open access article under the CC BY-NC-ND license (http://creativecommons.org/licenses/by-nc-nd/4.0/). 2666-061X/201507

https://doi.org/10.1016/j.asmr.2021.01.002 mineral density (BMD) T-score of -2.5 or less defines osteoporosis, whereas $\mathrm{T}$-scores between -2.5 and -1 define osteopenia. ${ }^{2}$ Rotator cuff (RC) tears are a common source of pain and dysfunction that predominantly affect the aging population. ${ }^{3}$ The structural integrity and healing of a repaired RC is important and has been associated with better outcomes. ${ }^{4}$ Several patient-specific factors have been reported to influence tendon healing after rotator cuff repair (RCR), including patient age, tear size, fatty infiltration, muscle atrophy, muscle retraction, smoking, diabetes, vitamin $\mathrm{D}$ deficiency, and osteoporosis. ${ }^{5-9}$ Furthermore, the greater tuberosity is known to become osteopenic after $\mathrm{RC}$ tears, which is correlated to the severity of retraction and chronicity of the tear. ${ }^{10-14}$ BMD has been shown to have significant effects on RCR healing, given that patients with osteopenia have a more than 4 -fold increased risk of repair failure and patients with osteoporosis have a 7 -fold increased risk of retear. ${ }^{2}$ Surgeons have recognized bone quality as an important prognostic factor for $\mathrm{RCR}^{15-17}$ and adjusted their 
technique by limiting decortication to rely on cortical and superficial trabecular bone for fixation, placing anchors $90 \underline{0}$ to the cortex, and distributing force with an increased number of anchors, suture tape, or advancement of the medial-row anchors. ${ }^{18,19}$ Animal models have suggested that bisphosphonates and teriparatide injections may increase the amount of stress the RC can withstand before failure. ${ }^{20,21}$

As of 2014, the U.S. National Osteoporosis Foundation (NOF) guidelines have included fragility fractures and fracture risk as estimated using the Fracture Risk Assessment Tool (FRAX) ${ }^{22}$ to define indications for pharmacologic treatment. ${ }^{23}$ To date, a paucity of literature exists examining whether patients undergoing RCR are being appropriately screened, as well as whether they are receiving pharmacologic management when indicated for low BMD. A previous study in a similar cohort reported that osteoporosis is common and undertreated in patients aged 48 to 92 years prior to total joint arthroplasty, with nearly $25 \%$ of patients meeting the NOF criteria for pharmacologic management. ${ }^{24}$

The purpose of this study was to (1) report the percentage of patients undergoing RCR who were appropriately screened with DXA testing prior to surgery, if indicated, and (2) determine the percentage of patients properly prescribed osteoporosis medications within 6 months of surgery. We hypothesized that preoperative osteoporosis would be under-screened in this population. However, in patients who were appropriately screened, we hypothesized that these patients would have received appropriate medical management.

\section{Methods}

Consecutive patients who underwent RCR at a single tertiary-care center over the year prior to study initiation (August 2018 to August 2019) were reviewed. Patients were identified by Current Procedural Terminology (CPT) codes for RCR: CPT code 29827 (arthroscopy, shoulder, surgical, with RCR), CPT code 23410 (repair of musculotendinous cuff, acute), CPT code 23412 (repair of musculotendinous cuff, chronic), and CPT code 23420 (reconstruction of complete shoulder [rotator] cuff avulsion, chronic [includes acromioplasty]). Patients were included if they were at least 50 years of age and underwent one of the aforementioned RCR procedures. The exclusion criteria included concomitant acute fractures (e.g., acute proximal humeral fracture undergoing hemiarthroplasty) or revision surgery. The indications for surgery, tendons repaired, and concomitant procedures were recorded from the surgeons' operative reports. Institutional review board exemption (under 45 CFR 46, category 4) was obtained for this study.

Electronic medical records were reviewed for demographic information, RC tear acuity (a tear was defined as acute if occurring within 3 months of surgical date), preoperative osteoporosis risk factors, previous low-energy adult-age fractures, secondary causes of osteoporosis, prior DXA testing, current calcium and vitamin D supplementation, and osteoporosis pharmacotherapy (prescription within 6 months before or after surgery).

\section{Fracture Risk Assessment}

The FRAX calculator is a fracture risk assessment tool intended for postmenopausal women and men aged 50 years or older to estimate the percentage risk of sustaining a fracture over a 10-year time frame. ${ }^{23}$ Separate percentages are determined for the risk of a hip fracture and the risk of all major osteoporotic fractures, defined as fractures of the hip, spine, wrist, or humerus. ${ }^{25}$ The FRAX calculator, recommended by NOF, was used to estimate fracture risk without femoral neck BMD for all patients. ${ }^{22}$ FRAX calculation is used as part of the diagnostic evaluation to determine which patients are indicated pharmacologic treatment of osteoporosis.

Patient risk factors included in the FRAX assessment are detailed in Table 1. A separate calculation of the FRAX percentage with femoral neck BMD was completed for patients who underwent DXA testing in the 2-year period preoperatively. The NOF criteria for BMD testing and pharmacologic osteoporosis treatment (Table 2) were applied to all patients.

\section{DXA Screening}

The lowest T-score from either the lumbar vertebrae (average of 2 lumbar vertebrae while excluding vertebrae with degenerative or surgical changes) or proximal femur (femoral neck and total femur) was recorded. An "appropriately screened" patient was defined as a patient who met the indications for BMD evaluation and underwent a DXA scan in the 2 years prior to RCR. An "appropriately treated" patient was defined as a patient who was receiving NOF-recommended and U.S. Food and Drug Administration-approved osteoporosis medications including bisphosphonates, estrogen agonist or antagonist (raloxifene), estrogen and/or hormone therapy, tissue-selective estrogen complex, parathyroid hormone 1-34 (teriparatide), and receptor activator of nuclear factor $\mathrm{KB}$ ligand inhibitor (denosumab). ${ }^{23}$ During the study period, there was no routine, standardized screening protocol used by providers and providerextenders for identifying patients at risk of osteoporosis and in need of further testing.

Statistical analysis was completed using IBM SPSS Statistics software (version 26; IBM, Armonk, NY). Shapiro-Wilk testing was performed to determine whether continuous variables were normally distributed. For parametric continuous variables, 2-sample $t$ tests were used, whereas Mann-Whitney $U$ testing was used for nonparametric variables. For categorical variables, $\chi^{2}$ analysis was performed. In some subsets, descriptive statistics were used because the intention 
Table 1. Clinical Risk Factors Included in FRAX ${ }^{22}$

Age

Sex

Body mass index

Previous fracture*

Hip fracture in parent

Active smoker

Glucocorticoid use

Diagnosis of rheumatoid arthritis

Secondary osteoporosis

$\geq 3$ Alcoholic drinks/d

Femoral neck BMD (in grams per square centimeter), if available

BMD, bone mineral density; FRAX, Fracture Risk Assessment Tool *Previous fracture in adult life occurring after low-energy trauma.

${ }^{\dagger}$ Equivalent to $5 \mathrm{mg}$ of prednisolone daily currently or for more than 3 months in the past.

${ }^{\ddagger}$ Secondary cause of osteoporosis: type 1 diabetes, osteogenesis imperfecta, untreated long-standing hyperthyroidism, hypogonadism or premature menopause, chronic malnutrition, or malabsorption and chronic liver disease.

was not to detect differences between groups but rather to profile the bone health of all patients presenting for RCR. $P<.05$ was considered statistically significant.

\section{Results}

A total of 218 patients aged at least 50 years underwent RCR during the aforementioned 1-year period. The mean age was $61.5 \pm 7.2$ years, and most patients were women $(\mathrm{n}=129,58.1 \%)$. The RC tears in most patients $(\mathrm{n}=131,60.1 \%)$ were acute (RCR within 3 months of injury). The vast majority of patients underwent RCR of the supraspinatus tendon (96.8\%) with subacromial decompression $(90.4 \%)$. A complete breakdown of patient demographic characteristics, surgical procedures performed, and osteoporosis risk factors is detailed in Table 3.

Adult-age fractures occurred in 25 patients (11.5\%), with 6 vertebral fractures $(2.8 \%), 5$ ankle fractures $(2.3 \%), 5$ distal radius fractures $(2.3 \%), 4$ proximal humeral fractures $(1.8 \%), 2$ tibial shaft fractures $(1 \%)$, 1 patellar fracture $(0.5 \%)$, I lateral compression pelvic fracture $(0.5 \%)$, and 1 hip fracture $(0.5 \%)$. Two patients had secondary causes of osteoporosis owing to malabsorption from long-standing inflammatory bowel disease. Table 4 details osteoporosis risk factors for the entire case series.

\section{Fracture Risk Assessment and Osteoporosis Screening}

A total of 69 patients $(31.7 \%)$ met the NOF criteria for BMD screening; however, only 23 of these 69 patients $(33.3 \%)$ were appropriately screened with a DXA scan within 2 years of the surgical date. A total of 50 patients $(22.9 \%)$ underwent a DXA scan at some point in their lifetime. The 23 patients who were appropriately screened underwent a DXA scan at a mean of $13.1 \pm 6.4$ months prior to the surgical date. The mean FRAX percentage for a major fracture in the next 10 years without BMD was $6.8 \% \pm 4.4 \%$ for the entire case series. Patients who were indicated for BMD screening but did not undergo a DXA scan had a FRAX major fracture risk of $10.9 \% \pm 5.5 \%$ and FRAX hip fracture risk of $2.3 \% \pm 2.2 \%$. These risk percentages were significantly higher $(P \leq .001)$ than those of patients not indicated for BMD screening. The latter group showed a FRAX major fracture risk of $4.9 \% \pm 1.9 \%$ and FRAX hip fracture risk of $0.5 \% \pm 0.4 \%$. Table 5 details the FRAX calculations for all subgroups.

The primary care provider (PCP) ordered the DXA scan in 18 of the 23 patients $(78.2 \%)$ who were appropriately screened with a DXA scan. An orthopaedic provider initiated the evaluation in 3 cases $(13.0 \%)$, an oncologist initiated the workup in 1 patient $(4.3 \%)$ because of a cancer history, and an obstetrics and gynecology provider initiated the workup in 1 patient $(4.3 \%)$ because this provider was serving in a PCP-type role. In 2 of the 3 cases in which an orthopaedic surgery provider ordered the DXA scan, the scan was obtained at the direction of our spine colleagues for patients with a fracture history whereas a single patient had a DXA scan ordered by a sports medicine provider after meeting the NOF screening criteria.

\section{Osteoporosis Population and Treatment}

Thirty-two patients $(14.7 \%)$ met the NOF criteria for pharmacologic management of osteoporosis based on

Table 2. NOF Guidelines for Which Patients Should Undergo BMD Screening and Receive Pharmacologic Therapy for Osteoporosis $^{23}$

\footnotetext{
BMD screening criteria

Women

All women aged $\geq 65 \mathrm{yr}$

Younger postmenopausal women and women in menopausal transition with clinical risk factors for fracture*

Women aged $\geq 50 \mathrm{yr}$ who have had adult-age fracture

Women with condition (e.g., rheumatoid arthritis) or taking medication (e.g., glucocorticoids, with daily dose of prednisone $\geq 5 \mathrm{mg}$ or equivalent for $\geq 3 \mathrm{mo}$ ) associated with low bone mass or bone loss medication (e.g., glucocorticoids, with daily dose of prednisone $\geq 5 \mathrm{mg}$ or equivalent for $\geq 3 \mathrm{mo}$ ) associated with low bone mass or bone loss

Guidelines for pharmacologic intervention

T-score $\leq 2.5$ at femoral neck or spine ${ }^{\dagger}$

History of hip or vertebral fracture

T-score between -2.5 and -1 at femoral neck or spine and $10-y r$ risk of hip fracture $\geq 3 \%$ or major osteoporotic fracture $\geq 20 \%$

BMD, bone mineral density; NOF, U.S. National Osteoporosis Foundation.

${ }^{*}$ Clinical risk factors found in Table 1 .

${ }^{\dagger}$ After appropriate evaluation to exclude secondary causes.
} 
Table 3. Patient Demographic Characteristics and Surgical Information

\begin{tabular}{|c|c|c|}
\hline & $\mathrm{n}$ or Mean & $\%$ or $\mathrm{SD}$ \\
\hline \multicolumn{3}{|l|}{$\overline{\operatorname{Sex}}$} \\
\hline Male & 89 & 40.8 \\
\hline Female & 129 & 59.2 \\
\hline Age, yr & 61.5 & 7.2 \\
\hline Body mass index & 31.1 & 6.5 \\
\hline \multicolumn{3}{|l|}{ Laterality } \\
\hline Right & 126 & 57.8 \\
\hline Left & 92 & 42.2 \\
\hline \multicolumn{3}{|l|}{ Ethnicity } \\
\hline White & 202 & 92.7 \\
\hline African American & 11 & 5.0 \\
\hline Hispanic or Latino & 2 & 0.9 \\
\hline Asian & 2 & 0.9 \\
\hline Native American & 1 & 0.5 \\
\hline Vitamin D level within $2 \mathrm{yr}$ of surgery & 48 & 22.0 \\
\hline Vitamin D deficiency & 17 & 7.8 \\
\hline Acute rotator cuff tear & 131 & 60.1 \\
\hline \multicolumn{3}{|l|}{ Surgical procedures performed } \\
\hline Supraspinatus repair & 211 & 96.8 \\
\hline Infraspinatus repair & 89 & 40.8 \\
\hline Subscapularis repair & 49 & 22.5 \\
\hline Biceps tenotomy & 113 & 51.8 \\
\hline Biceps tenodesis & 42 & 19.3 \\
\hline $\begin{array}{l}\text { Mumford procedure or distal clavicle } \\
\text { excision }\end{array}$ & 103 & 47.2 \\
\hline $\begin{array}{l}\text { Subacromial decompression with or } \\
\text { without acromioplasty }\end{array}$ & 197 & 90.4 \\
\hline
\end{tabular}

either DXA scan, FRAX calculation, or the presence of an osteoporotic fracture. This population consisted of 14 of the 23 patients $(60.9 \%)$ who were indicated based on DXA scan results, 6 patients who had vertebral fractures and another patient who had a hip fracture, and 18 patients who had either a FRAX major fracture risk greater than $20 \%$ or a FRAX hip fracture risk greater than $3 \%$ in the next 10 years. In 6 of the 32 patients $(18.8 \%)$, more than 1 parameter indicating pharmacologic management was met. Of the 32 patients meeting the medication criteria, only 5 (15.6\%) were prescribed an appropriate medication. Three patients were prescribed teriparatide injections, and two were prescribed a bisphosphonate medication. Fig 1 details the screening and pharmacologic management of the study cohort. Patients meeting the criteria for treatment with medication were, on average, significantly older $(69.1 \pm 7.7$ years vs $60.2 \pm 6.3$ years, $P<.001)$ and had a significantly lower BMI $(28.8 \pm 6.1$ vs $31.5 \pm 6.5, P=.028$ ). These patients were also more frequently found to have chronic RC tears $(P=.015)$.

\section{Discussion}

The findings of this study demonstrate that approximately one-third of patients aged 50 years or older undergoing RCR meet the criteria for osteoporosis screening, although only $33.3 \%$ of these patients are appropriately screened. In addition, $14.7 \%$ of all patients meet the criteria for osteoporosis medication, but only $15.6 \%$ of these patients receive appropriate pharmacologic therapies. In most of the patients who were appropriately screened, their PCP performed screening. Patients with low BMD meeting the criteria for treatment with medication were nearly 9 years older, on average; had a lower body mass index; and had chronic RC tears.

Osteoporosis negatively affects clinical outcomes in patients undergoing RCR. Chung et al. ${ }^{2}$ evaluated postoperative RCR integrity in 272 patients with a mean age of 59.5 years and identified osteoporosis as an independent risk factor for failure on multivariate analysis after controlling for patient age. Nho et al. ${ }^{26}$ noted that older patients have 1.08 times greater odds of a tendon defect after repair with each 1 -year increase in age. In a cohort study using the PearlDiver database (PearlDiver, Colorado Springs, CO) including 2,706 patients who underwent arthroscopic RCR, it was reported that patients with osteoporosis had a higher rate of revision RCR $(6.58 \%)$ than patients without osteoporosis $(4.51 \%, P=.008)$. Yet, patients with a diagnosis of osteoporosis who were appropriately prescribed bisphosphonates did not have significantly lower revision RCR rates as compared with age- and sex-matched patients with osteoporosis not receiving bisphosphonates. ${ }^{27}$ These results suggest that bisphosphonate therapy in the patient with osteoporosis and an RC tear may not necessarily lead to improvement in clinical outcomes. Teriparatide has been shown in an animal model to increase bone density of the humeral head, and it may improve the mechanical properties of the infraspinatus tendon enthesis. ${ }^{21}$ Further study of this and other medications used to treat osteoporosis will be needed to determine their effect on RCR outcomes.

Despite the importance of BMD to the clinical outcomes of RCR, it may not be advisable to delay surgery to improve BMD pharmacologically in the setting of an acute RC tear. Acute RC tears have been shown to have superior clinical outcomes if repaired early. ${ }^{6,28-31}$ In addition, it may take several months or longer to significantly improve BMD with pharmacologic intervention. ${ }^{32-34}$ In patients with

Table 4. Osteoporosis Risk Factors for All Patients $(\mathrm{N}=218)$

\begin{tabular}{lrr}
\hline & $\mathrm{n}$ & \multicolumn{1}{c}{$\%$} \\
\hline Previous fracture at age $\geq 50 \mathrm{yr}$ & 25 & 11.5 \\
Fracture in lifetime & 48 & 22.1 \\
Previous hip fracture & 1 & 0.5 \\
Previous spine fracture & 6 & 2.8 \\
Hip fracture in parent & 3 & 1.4 \\
Active smoker & 35 & 16.1 \\
Glucocorticoid use & 11 & 5.0 \\
Rheumatoid arthritis & 1 & 0.5 \\
3 Alcoholic drinks/d & 12 & 5.5 \\
Secondary osteoporosis & 2 & 0.9 \\
\hline
\end{tabular}


Table 5. FRAX Calculations for Series and Subgroups

\begin{tabular}{lcc}
\hline & \multirow{2}{*}{ FRAX Calculation, \% } \\
\cline { 2 - 3 } & Mean & SD \\
\hline Entire series without BMD $(\mathrm{N}=218)$ & 6.8 & 4.4 \\
$\quad$ Major fracture risk & 1.1 & 1.6 \\
$\quad$ Hip fracture risk & & \\
Patients not meeting screening criteria & & \\
$\quad(\mathrm{n}=149)$ & 4.9 & 1.9 \\
$\quad$ Major fracture risk & 0.5 & 0.4 \\
$\quad$ Hip fracture risk & & \\
Patients meeting screening criteria $(\mathrm{n}=69)$ & 10.9 & 5.5 \\
$\quad$ Major fracture risk & 2.3 & 2.2 \\
$\quad$ Hip fracture risk & & \\
Patients with BMD at femoral neck $(\mathrm{n}=23)$ & 11 & 5.0 \\
$\quad$ Major fracture risk & 1 & 0.5 \\
$\quad$ Hip fracture risk & & \\
\hline
\end{tabular}

NOTE. FRAX percentages are listed for the case series as a whole and for patients who were indicated for dual-energy X-ray absorptiometry (DXA) testing but did not receive testing, patients who were indicated and did receive DXA testing, and patients who underwent a DXA scan within 2 years of surgery.

BMD, bone mineral density; FRAX, Fracture Risk Assessment Tool; $\mathrm{SD}$, standard deviation.

chronic tears or tears amenable to initial nonoperative management, it may be reasonable to screen for low BMD and begin treatment. Orthopaedic surgeons may be the first providers to see these patients and should be familiar with the screening criteria for osteoporosis. The importance of early detection and screening cannot be understated, especially if patients will require eventual surgical management. Our study identified that PCPs are often the providers ordering DXA scans in patients with RC tears who meet indications. At least in the study population at our institution, orthopaedic surgeons rarely were the providers initiating bone health evaluation. Furthermore, 46 patients $(21.1 \%)$ were not appropriately screened despite presenting for at least 1 orthopaedic office visit prior to surgery. Although these findings may be specific to this patient population, they highlight the need for further education of orthopaedic providers.

Diagnosis of an RC tear represents an opportunity to improve overall patient health. Despite the significant morbidity and mortality of fragility fractures, the United States continues to be inconsistent with recommended screening and treatment, leading to what some experts have labeled a bone health "crisis." ${ }^{\text {35-38 }}$ This has been recognized as a critical issue by the American Orthopaedic Association, leading to the development of the Own the Bone (OTB) initiative. ${ }^{37}$ As stated by the OTB program, all patients aged 50 years or older scheduled to undergo major orthopaedic surgery should be screened for low BMD. ${ }^{37}$ The OTB program, which launched in 2009, includes a Web-based registry program to empower medical systems to reduce the incidence of fractures and initiate early, appropriate medical management. ${ }^{39}$ This initiative also emphasizes the critical role and responsibility orthopaedic surgeons have in recognizing the need for screening and initiating referrals. Our institution created a fracture liaison service and metabolic bone clinic staffed by physicians and physician-extenders with intimate knowledge of screening guidelines, as well as indications, duration, and dosing of pharmacologic treatment including but not limited to calcium and vitamin D supplementation, activity recommendations, and both anabolic and antiresorptive medications. Surgeons can refer patients to this clinic for prompt and comprehensive bone health optimization.

Despite these programs being in place at the study institution, a significant percentage of patients were not appropriately screened based on the NOF and OTB guidelines. The reasons are likely multifactorial. First,

Fig 1. Percentages of patients who met U.S. National Osteoporosis Foundation (NOF) criteria for bone mineral density (BMD) screening and number of patients appropriately screened, along with number of patients who met criteria for osteoporosis pharmacologic management and number of patients indicated for medication who were prescribed medication.

\section{Screening and Treatment of Osteoporosis in Rotator Cuff Repair Patients ( $\mathrm{N}=\mathbf{2 1 8}$ )}

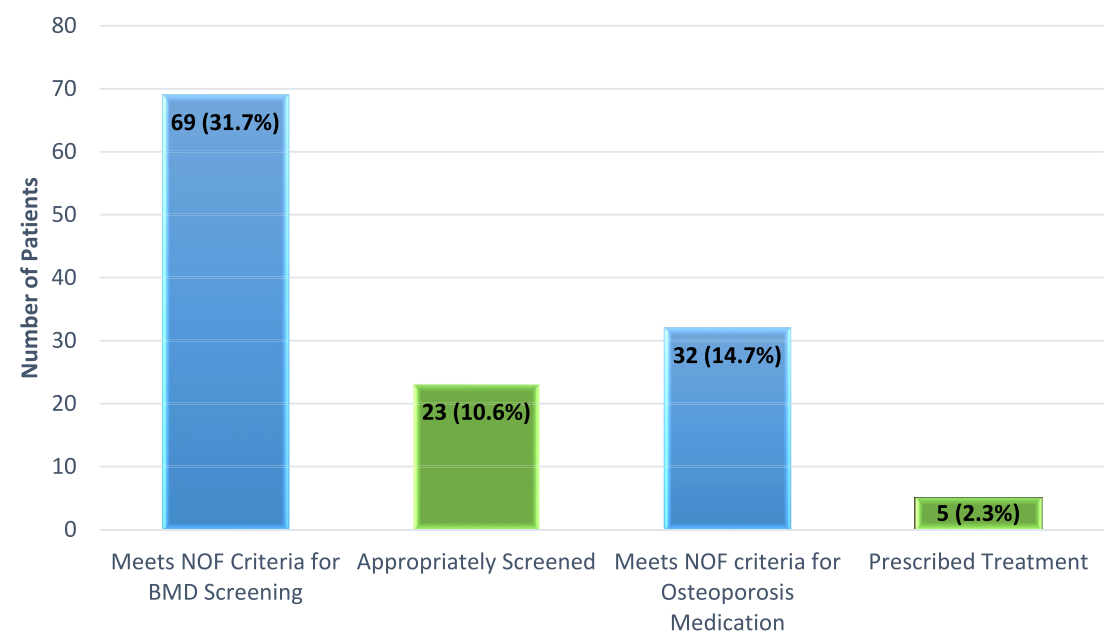


consultations with the fracture liaison service at our center are triggered by a sentinel event, namely a lowenergy mechanism fracture. If a patient does not sustain a fracture, then this service is not typically consulted. Another potential reason is lack of understanding of the NOF screening guidelines by both PCPs and orthopaedic surgeons. Although the OTB program has done an excellent job of raising awareness through initiatives, guidelines, and publications, the screening criteria have not been integrated into a new patient checklist or other part of the clinic workflow, reminding busy surgeons and trainees in the clinic to investigate bone health. A final reason postulated for the low screening rates is that bone health may not have been seen as a significant factor affecting clinical outcomes and operative techniques within the orthopaedic-sports medicine department in comparison with subspecialties such as trauma, spine, and adult reconstruction. Given the results of this study, expansion of the institution's fracture liaison service to capture at-risk patients undergoing any elective surgical procedure would be beneficial. ${ }^{37,40}$ Ongoing quality-improvement efforts are underway to address this issue, including placement of the NOF screening criteria on the walls of provider workrooms in the clinic both as a quick reference and as a reminder to diligently assess bone health when reviewing the medical history with the patient. Ideally, this will be integrated into the electronic medical record as part of a new patient intake process.

\section{Limitations}

The retrospective nature of the study design is limited by the accuracy of recorded data in the medical record. At the time of data collection, there was no formal osteoporosis screening protocol in place within the sports medicine department; thus, these data may not be reflective of other institutions with screening protocols. Multiple surgeons contributed cases to this series, and there may be variation in surgical indications and approaches to bone health screening based on individual surgeon preferences. Furthermore, this study did not directly examine any clinical outcomes of RCR and the effects of bone health on outcome. Finally, these data are reflective of the patient population seen in a single geographic region of the United States. It is not known if these data are reflective of patient populations in other regions.

\section{Conclusions}

Patients aged 50 years or older undergoing RCR are often not appropriately screened for osteoporosis. Even when appropriately screened, only $15.6 \%$ of patients meeting the indications for pharmacologic intervention for bone health optimization were prescribed appropriate medications. Although bone health optimization may or may not affect surgical timing, patient encounters related to RC tears can be used as an opportunity for providers to initiate osteoporosis screening and treatment protocols.

\section{References}

1. Wright NC, Looker AC, Saag KG, et al. The recent prevalence of osteoporosis and low bone mass in the United States based on bone mineral density at the femoral neck or lumbar spine. J Bone Miner Res 2014;29:2520-2526.

2. Chung SW, Oh JH, Gong HS, Kim JY, Kim SH. Factors affecting rotator cuff healing after arthroscopic repair: Osteoporosis as one of the independent risk factors. Am J Sports Med 2011;39:2099-2107.

3. Neer CS II, Craig EV, Fukuda H. Cuff-tear arthropathy. J Bone Joint Surg Am 1983;65:1232-1244.

4. Slabaugh M, Nho S, Grumet R, et al. Does the literature confirm superior clinical results in radiographically healed rotator cuffs after rotator cuff repair? Arthroscopy 2010;26: 393-403.

5. Abtahi AM, Granger EK, Tashjian RZ. Factors affecting healing after arthroscopic rotator cuff repair. World $J$ Orthop 2015;6:211-220.

6. Mall NA, Tanaka MJ, Choi LS, Paletta GA. Factors affecting rotator cuff healing. J Bone Joint Surg Am 2014;96:778-788.

7. Charousset C, Bellaïche L, Kalra K, Petrover D. Arthroscopic repair of full-thickness rotator cuff tears: Is there tendon healing in patients aged 65 years or older? Arthroscopy 2010;26:302-309.

8. Angeline ME, Ma R, Pascual-Garrido C, et al. Effect of diet-induced vitamin D deficiency on rotator cuff healing in a rat model. Am J Sports Med 2014;42:27-34.

9. Almeida A, Atti V, Agostini DC, Valin MR, de Almeida NC, Agostini AP. Comparative analysis on arthroscopic sutures of large and extensive rotator cuff injuries in relation to the degree of osteopenia. Rev Bras Ortop 2015;50:83-88.

10. Cadet ER, Hsu JW, Levine WN, LU Bigliani, Ahmad CS, York N. The relationship between greater tuberosity osteopenia and the chronicity of rotator cuff tears. J Shoulder Elbow Surg 2008;17:73-77.

11. Oh JH, Song BW, Kim SH, et al. The measurement of bone mineral density of bilateral proximal humeri using DXA in patients with unilateral rotator cuff tear. Osteoporos Int 2014;25:2639-2648.

12. Kannus P, Leppälä J, Lehto M, Sievänen H, Heinonen A, Järvinen M. A rotator cuff rupture produces permanent osteoporosis in the affected extremity, but not in those with whom shoulder function has returned to normal. J Bone Miner Res 1995;10:1263-1271.

13. Waldorff EI, Lindner J, Kijek TG, et al. Bone density of the greater tuberosity is decreased in rotator cuff disease with and without full-thickness tears. J Shoulder Elbow Surg 2011;20:904-908.

14. Galatz LM, Sandell LJ, Rothermich SY, et al. Characteristics of the rat supraspinatus tendon during tendon-tobone healing after acute injury. J Orthop Res 2006;24: 541-550.

15. Kirchhoff C, Braunstein V, Milz S, et al. Assessment of bone quality within the tuberosities of the osteoporotic humeral head. Am J Sports Med 2010;38:564-569. 
16. Barber FA, Feder SM, Burkhart SS, Ahrens J. The relationship of suture anchor failure and bone density to proximal humerus location: A cadaveric study. Arthroscopy 1997; 13:340-345.

17. Tingart MJ, Apreleva M, Zurakowski D, Warner JJP. Pullout strength of suture anchors used in rotator cuff repair. J Bone Joint Surg Am 2003;85:2190-2988.

18. Entezari V, Lazarus M. Surgical considerations in managing osteoporosis, osteopenia, and vitamin D deficiency during arthroscopic rotator cuff repair. Orthop Clin North Am 2019;50:233-243.

19. Denard PJ, Burkhart SS. Techniques for managing poor quality tissue and bone during arthroscopic rotator cuff repair. Arthroscopy 2011;27:1409-1421.

20. Cadet ER, Vorys GC, Rahman R, et al. Improving bone density at the rotator cuff footprint increases supraspinatus tendon failure stress in a rat model. J Orthop Res 2010;28:308-314.

21. Chen X, Giambini H, Ben-Abraham E, An KN, Nassr A, Zhao C. Effect of bone mineral density on rotator cuff tear: An osteoporotic rabbit model. PLoS One 2015;10:e0139384.

22. Centre for Metabolic Bone Diseases, University of Sheffield. FRAX: Fracture Risk Assessment Tool, https://www. sheffield.ac.uk/FRAX/tool.aspx? country=9. Accessed March 21, 2020.

23. Cosman F, De Beur SJ, Leboff MS, et al. Clinician's guide to prevention and treatment of osteoporosis. Osteoporos Int 2014;25:2359-2381.

24. Bernatz JT, Brooks AE, Squire MW, Illgen RI, Binkley NC, Anderson PA. Primary arthroplasty osteoporosis is common and undertreated prior to total joint arthroplasty. J Arthroplasty 2019;34:1347-1353.

25. Briot K, Paternotte S, Kolta S, et al. FRAX(R): Prediction of major osteoporotic fractures in women from the general population: The OPUS study. PLoS One 2013;8: e83436.

26. Nho SJ, Brown BS, Lyman S, Adler RS, Altchek DW, Macgillivray JD. Prospective analysis of arthroscopic rotator cuff repair: Prognostic factors affecting clinical and ultrasound outcome. J Shoulder Elbow Surg 2009;18:13-20.

27. Cancienne JM, Brockmeier SF, Kew ME, Deasey MJ, Werner BC. The association of osteoporosis and bisphosphonate use with revision shoulder surgery after rotator cuff repair. Arthroscopy 2019;35:2314-2320.

28. Duncan NS, Booker SJ, Gooding BWT, Geoghegan J, Wallace WA, Manning PA. Surgery within 6 months of an acute rotator cuff tear significantly improves outcome. J Shoulder Elbow Surg 2015;24:1876-1880.

29. Spross C, Behrens G, Dietrich TJ, et al. Early arthroscopic repair of acute traumatic massive rotator cuff tears leads to reliable reversal of pseudoparesis: Clinical and radiographic outcome. Arthroscopy 2019;35:343-350.

30. Hantes ME, Karidakis GK, Vlychou M, Varitimidis S, Dailiana Z, Malizos KN. A comparison of early versus delayed repair of traumatic rotator cuff tears. Knee Surg Sports Traumatol Arthrosc 2011;19:1766-1770.

31. Petersen SA, Murphy TP. The timing of rotator cuff repair for the restoration of function. J Shoulder Elbow Surg 2011;20:62-68.

32. Suzuki T, Sukezaki F, Shibuki T, Toyoshima Y, Nagai T, Inagaki K. Teriparatide administration increases periprosthetic bone mineral density after total knee arthroplasty: A prospective study. J Arthroplasty 2018;33:79-85.

33. Lin T, Yan S-G, Cai X-Z, Ying Z-M. Bisphosphonates for periprosthetic bone loss after joint arthroplasty: A metaanalysis of 14 randomized controlled trials. Osteoporos Int 2012;23:1823-1834.

34. Bhandari M, Bajammal S, Guyatt GH, et al. Effect of bisphosphonates on periprosthetic bone mineral density after total joint arthroplasty. A meta-analysis. J Bone Joint Surg Am 2005;87:293-301.

35. Morin S, Lix LM, Azimaee M, Metge C, Caetano P, Leslie WD. Mortality rates after incident non-traumatic fractures in older men and women. Osteoporos Int $2011 ; 22: 2439-2448$.

36. Leslie WD, Giangregorio LM, Yogendran M, et al. A population-based analysis of the post-fracture care gap 1996-2008: The situation is not improving. Osteoporos Int 2012;23:1623-1629.

37. Anderson PA, Jeray KJ, Lane JM, Binkley NC. Bone health optimization: Beyond Own the Bone: AOA critical issues. J Bone Joint Surg Am 2019;101:1413-1419.

38. Khosla S, Shane E. A crisis in the treatment of osteoporosis. J Bone Miner Res 2016;31:1485-1487.

39. American Orthopaedic Association. Own the Bone, https://www.ownthebone.org/. Accessed March 21, 2020.

40. Beaton DE, Vidmar M, Pitzul KB, et al. Addition of a fracture risk assessment to a coordinator's role improved treatment rates within 6 months of screening in a fragility fracture screening program. Osteoporos Int 2017;28: 863-869. 